\title{
Data Conflicts: the Dwindling Power of a Study
}

\author{
Debajyoti Mohanty
}

Received: 7 July 2014 / Accepted: 22 July 2014 / Published online: 3 August 2014

(C) Association of Surgeons of India 2014

I have read the article "Consequences of spilled gallstones during laparoscopic cholecystectomy (LC)" by Suvi Virupaksha [1] with interest. The unpredictable outcome of spilled gallstones that are left un-retrieved during LC is often a cause of future embarrassment for the operating surgeon. So, it comes as a surprise that most instances of unrecovered spilled gallstones are neither documented in the operation notes nor disclosed to the patients.

This prospective observational study was carried out in 150 (1 gallbladder polyp, 2 acalculous cholecystitis, and 147 gallstones) patients of LC over a period of one year. There are many glaring inconsistencies in the presented data that merit clarification by the author. The table outlining the ultrasound findings mentioned of 10 patients with solitary gallstone while multiple stones were detected in 135 patients. This amounts to a total of 145 instead of 147 patients with gallstone disease. Similarly, the sum total of patients of the three different groups categorized on the basis of stone size is 156 instead of 147. The author has variably reported the incidence of gallstone spillage in this article $(19.04 \%$ in the text and $18.91 \%$ in the table). All the instances of gallstone spillage in this series were secondary to gallbladder perforations, but in the table comparing the results of the present study with international data, the reported incidence of spillage is more than that of gallbladder perforation. The various mechanisms responsible for gallbladder perforation are detailed in a tabular form; however, the numbers mentioned against different mechanisms are quite confusing as they are neither adding up

D. Mohanty $(\bowtie)$

Department of General Surgery, All India Institute of Medical

Sciences, Raipur, Chhattisgarh 492099, India

e-mail: debajyoti.mohanty@gmail.com to the total number of patients nor to the percentage of patients with spilled stones. The author has boasted of zero percent incidence of un-retrieved gallstones, but at the same time, mentioned of a patient with port-site wound complication secondary to a left out gallstone in the subcutaneous plane.

The author could have analyzed the incidence of spilled stones in relation to the incidence of complicated gallstone disease, aberrant ultrasound findings, and abnormal histopathological reports to predict risk factors for gallbladder perforation and stone spillage. Mention of any special effort made to locate and retrieve the spilled stones during LC, any change in the duration of postoperative antibiotics to counteract the increased risk of infective complications consequent to intraoperative spillage of bile and stones would have been immensely beneficial to the numerous readers keen on mastering the art of this common laparoscopic procedure.

The author has summarized the study with a list of suggestions to reduce the rate of stone spillage during LC. Few other additions to this list can be as follows: secure grasping of the neck of the excised gallbladder along with the cystic duct clip during extraction, adequate enlargement of the port site for smooth extrication followed by meticulous examination of the retrieved specimen for integrity of the gallbladder wall. Any defect in the wall should prompt repeat inspection of all the relevant intra-peritoneal recesses as well as the path of extrication of the gallbladder.

\section{Reference}

1. Virupaksha S (2014) Consequences of split gallstones during laparoscopic cholecystectomy. Indian J Surg 76(2):95-99 